\title{
Eritrodermia psoriática en un hombre joven: sospechar infección por VIH
}

\author{
Javier Arellano, Mathias Yagnam, Martín Vidal y Yamile Corredoira
}

\section{Erythrodermic psoriasis in young man: suspect HIV infection}

HIV infection can be manifested with different skin symptoms, which are sometimes considered infection markers. Erythrodermic psoriasis is a possible manifestation, which is a widespread form of psoriasis. We report a clinical case of a young man suspected of HIV infection due to a psoriatic erythroderma confirmed by biopsies, associated with Kaposi sarcoma. Afterwards, HIV infection was confirmed by serological tests. Antiretroviral therapy was started, with positive response at one month of treatment. Erythrodermic psoriasis can be considered a skin marker of HIV infection when occurs in previously healthy patients or in recalcitrant psoriasis.

Key words: HIV; dermatology; erythroderma; psoriasis; skin.

Palabras clave: VIH; dermatología; eritrodermia; psoriasis; piel.

\section{Introducción}

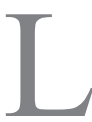

a pandemia del VIH continúa siendo un problema de salud en Chile y en el mundo. Se estima que existen 36,7 millones de personas infectadas al año 2015, y que durante ese año se produjeron 2,1 millones de infecciones nuevas en el mundo ${ }^{1}$. En Chile se había mantenido una tasa de notificación relativamente estable al año $2012^{2}$.

Las manifestaciones cutáneas de los pacientes infectados por VIH aparecen hasta en $94 \%$ de los $\operatorname{casos}^{3}$, siendo su incidencia proporcional a mayor inmunocompromiso ${ }^{4}$. Estas manifestaciones se deben a alteraciones tempranas en el sistema inmune de la piel, dentro de las que se incluyen la disminución del recuento de los linfocitos T CD4 y de la relación de los linfocitos T CD4/CD8, la pérdida de células dendríticas presentadoras de antígenos, el desbalance de la respuesta de linfocitos T helper (Th), con predominancia de Th2 y cambios en concentración de ciertas citoquinas, entre otras alteraciones ${ }^{5-8}$.

Las manifestaciones de la piel se pueden clasificar en infecciosas y no infecciosas. Dentro de las no causadas por infección se incluye la psoriasis, que en pacientes infectados por VIH tiende a ser más intensa y recalcitrante, y con una mayor frecuencia de artritis psoriática ${ }^{6}$.

La infección por VIH puede gatillar una eritrodermia psoriática9 ${ }^{9}$ La eritrodermia o dermatitis exfoliativa es una dermatopatía inflamatoria definida como un eritema y descamación de la piel que compromete más de $90 \%$ de la superficie corporal total ${ }^{10}$. Las dermatosis preexistentes son la principal causa de la eritrodermia (52\% del total de eritrodermias), siendo la psoriasis la más frecuente en adultos $(23 \% \text { de los casos })^{11}$ (Tabla 1$)$.
El siguiente caso clínico tiene por objetivo enfatizar la importancia de los marcadores cutáneos en la infección por VIH, como también, revisar cuáles son las mejores alternativas terapéuticas en pacientes con infección por VIH con psoriasis asociada.

\section{Caso clínico}

Varón de 31 años de edad, sin antecedentes médicos de importancia. Consultó por un cuadro de siete meses de evolución caracterizado por lesiones papulares en piernas, dorso, manos y pies, las cuales se extendieron a casi la totalidad del cuerpo, asociado a dolor articular, sin fiebre ni prurito. Al examen físico presentaba una eritrodermia con lesiones eritematosas infiltrativas con leve descamación, formando placas, respetando la cara y pliegues. Además, presentaba una queratodermia palmo-plantar de color amarillenta y lesiones en placa de coloración violácea en el dorso y tórax (Figura 1 y 2). Se realizaron biopsias cutáneas de las lesiones, cuyos hallazgos fueron compatibles con una psoriasis y un sarcoma de Kaposi. El estudio histológico demostró una agranulosis, adelgazamiento suprapapilar, paraqueratosis con polimorfonucleares intracórneos evidentes y vasos capilares prominentes en la dermis papilar, hallazgos compatibles con una dermatitis perivascular superficial psoriasiforme (Figura 3). Dada la presentación clínica de una eritrodermia psoriática, sin otros antecedentes, asociado a un sarcoma de Kaposi, se solicitaron pruebas serológicas para infección por VIH, además de exámenes para evaluar compromiso sistémico. Se internó con indicación sólo de lubricación cutánea en espera de los resultados, los que fueron reactivos para
Hospital Clínico San BorjaArriarán. Santiago, Chile. Facultad de Medicina, sede Centro, Universidad de Chile. Servicio de Dermatología (JA, MY).

Servicio de Anatomía Patológica (YC).

Universidad Finis Terrae. Santiago, Chile. Programa de Internado de Medicina (MV).

Sin financiamiento ni conflictos de interés.

Recibido: 8 de abril de 2017 Aceptado: 10 de octubre de 2017

Correspondencia a: Mathias Yagnam myagnamd@gmail.com 


\section{Tabla 1. Posibles gatillantes de la eritrodermia psoriática*}

Medicamentos (ej: terbinafina, litio, antimaláricos)

Irritantes tópicos (ej: coal tar)

Suspensión brusca de tratamientos (ej: corticoesteroides, metotrexato, biológicos)

Enfermedades sistémicas

Infecciones (ej: VIH)

Embarazo

Estrés

Quemaduras por fototerapia

* Adaptado de referencia 11.

\begin{tabular}{|c|c|c|}
\hline Fármaco & Dosis & Vía de administración \\
\hline Ciclosporina & 4-5 mg/kg al día & Oral \\
\hline Metotrexato & $\begin{array}{l}\text { 5-25 mg semanal según función renal y } \\
\text { respuesta clínica }\end{array}$ & Oral \\
\hline Acitretina & 25-50 mg al día & Oral \\
\hline Micofenolato mofetilo & 1-3 g al día & Oral \\
\hline Infliximab & 5-10 mg al día & Intravenoso \\
\hline Etanercept & $25 \mathrm{mg}$, dos veces a la semana & Subcutáneo \\
\hline Metotrexato + Infliximab & $\begin{array}{l}\text { 5-7,5 mg semanal de metotrexato }+ \\
2,7-4,4 \mathrm{mg} / \mathrm{gg} \text { de Infliximab }\end{array}$ & Oral e intravenoso \\
\hline Acitretina + Infliximab & $\begin{array}{l}0,3-0,6 \mathrm{mg} / \mathrm{kg} \text { de acitretina }+5 \mathrm{mg} / \mathrm{kg} \\
\text { de infliximab }\end{array}$ & Oral e intravenoso \\
\hline Ciclosporina + Etretinato & $\begin{array}{l}3,5-4 \mathrm{mg} / \mathrm{kg} \text { al día de ciclosporina }+ \\
0,5-0,6 \mathrm{mg} / \mathrm{kg} \text { al día de etretinato }\end{array}$ & Oral \\
\hline
\end{tabular}

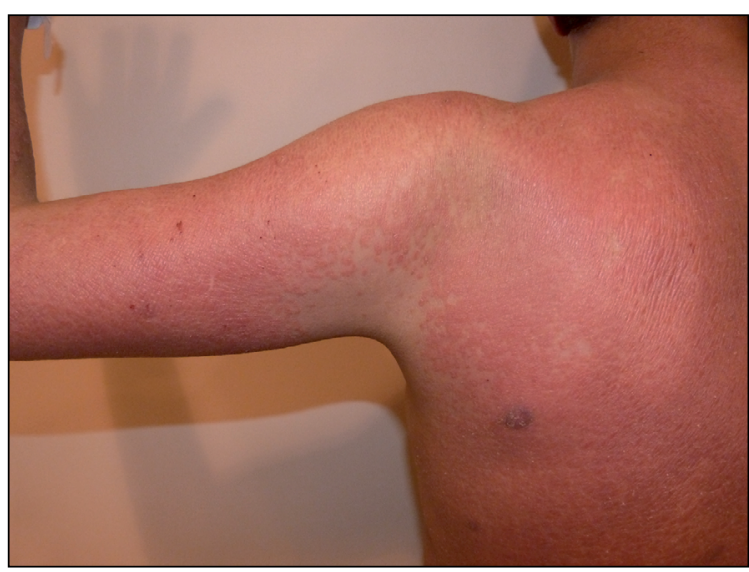

Figura 1. Múltiples placas eritematosas descamativas confluentes en todo el tronco.

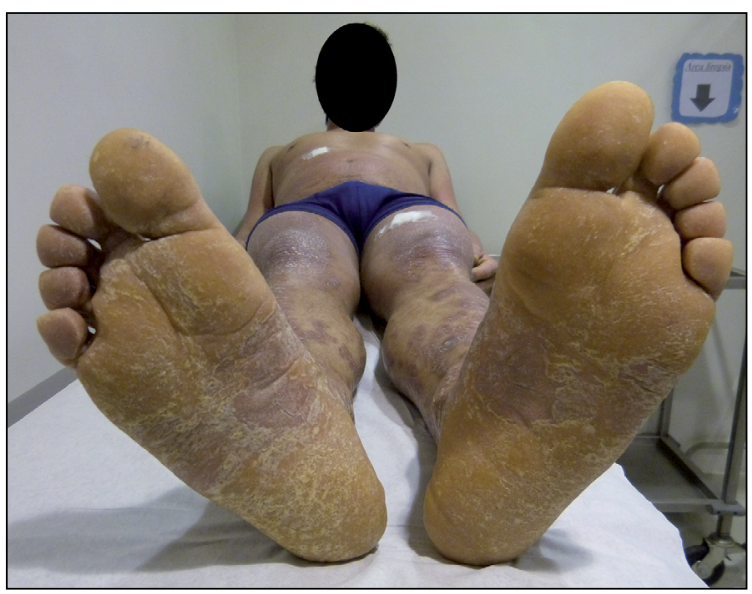

Figura 2. Queratodermia plantar y múltiples placas eritematosas descamativas en extremidades y tronco.
Figura 3. Biopsia de piel. A: Hiperplasia psoriasiforme regular epidérmica con gran escama paraqueratósica (40x). B: Paraqueratosis con neutrófilos intracórneos, agranulosis, adelgazamiento suprapapilar de la epidermis y vasos capilares prominentes en la dermis papilar (200x).

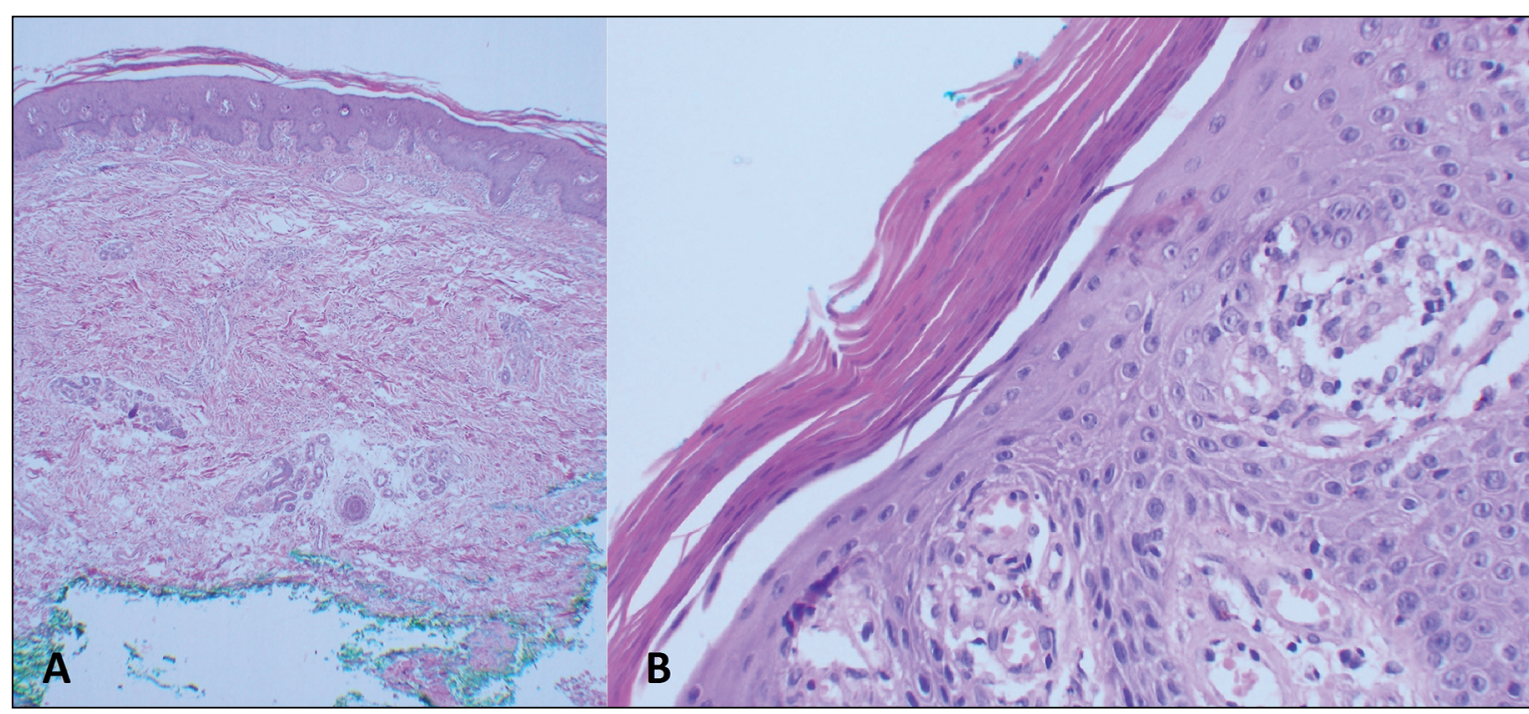

Rev Chilena Infectol 2017; 34 (6): 603-606 
VIH (etapa SIDA). El paciente se manejó con terapia antiretroviral, evolucionando favorablemente, con muy buena respuesta y resolución de gran porcentaje de las lesiones en un mes.

\section{Discusión}

La psoriasis tiene una prevalencia de $2-3 \%$ en la población general, siendo similar en los pacientes infectados con VIH; sin embargo, en estos últimos, la enfermedad tiende a presentarse en personas más jóvenes, sin antecedentes personales ni familiares de psoriasis, con una presentación clínica más agresiva, de predominio acral, con mayor frecuencia de artritis psoriática (hasta $50 \%$ de los casos) y peor respuesta al tratamiento ${ }^{7,12}$. La forma clínica más frecuente en pacientes infectados con VIH es la psoriasis en placas (78\%), seguido de la psoriasis inversa (37\%), guttata (29\%) y palmo-plantar $(8 \%)^{13}$. Sin embargo, esto varía según las series, tal es el caso de un estudio sudafricano, donde la forma clínica más frecuente fue la eritrodérmica ${ }^{14}$. Esto podría deberse a la consulta tardía, con la enfermedad en estadios más avanzados. Una característica distintiva de la psoriasis en pacientes infectados con VIH es la coexistencia simultánea de diferentes formas clínicas o a través del tiempo. En estos pacientes, $25 \%$ cumple criterios de psoriasis grave y tienen mayor riesgo de sobreinfección ${ }^{13,14}$.

Se describen dos grupos de pacientes con psoriasis e infección por VIH: el primero (un tercio de los casos) con psoriasis previa a la infección por VIH, presentan diagnóstico a edades más precoces y tienen antecedentes familiares y personales de psoriasis. El segundo grupo (dos tercios de los casos) presenta psoriasis posterior a la infección (en promedio cinco años después), con una menor proporción de antecedentes familiares y mayor frecuencia de compromiso palmo-plantar, inverso y artropatía ${ }^{15}$. Ambos grupos pueden presentarse como una eritrodermia psoriática ${ }^{4}$. Nuestro paciente se encuentra en el segundo grupo, debido a la aparición de una psoriasis posterior a la infección por VIH, falta de antecedentes familiares, y por presentar una queratodermia palmoplantar y artropatía.

En Latinoamérica, hay escasos reportes de casos de psoriasis en pacientes con VIH. La serie más extensa es de nueve casos, de los cuales ocho eran hombres, la edad promedio fue de 34 años y la localización más frecuente de las lesiones fue en la cara, pliegues y zona acral. El diagnóstico de psoriasis en ocho casos fue posterior a la infección por VIH. Uno de los casos presentó una eritrodermia psoriática y dos presentaron compromiso $\operatorname{articular}^{16}$. González y cols., en Cuba describen dos pacientes con psoriasis guttata e infección por VIH, uno de los cuales presentó el diagnóstico de una psoriasis posterior al diagnóstico de VIH y otro con psoriasis previa a la infección ${ }^{17}$.

La psoriasis es la segunda causa de eritrodermia en los pacientes infectados con VIH, luego de las reacciones adversas a medicamentos ${ }^{18}$. Entre las características clínicas de la eritrodermia psoriática están la presencia de placas bien circunscritas en los márgenes de la eritrodermia y alteraciones típicas de psoriasis ungueal como pits, manchas de aceite, onicolisis e hiperqueratosis subungueal ${ }^{19}$.

Dos tercios de los pacientes con eritrodermia presentan alteraciones histológicas sugerentes de alguna etiología ${ }^{20}$. De las histologías compatibles con eritrodermia psoriática, $60 \%$ presenta hallazgos histológicos de psoriasis inicial, $30 \%$ de placa psoriática bien desarrollada y $10 \%$ de psoriasis en regresión ${ }^{21}$. El paciente del caso presentó hallazgos histológicos de psoriasis inicial.

La eritrodermia psoriática se puede considerar un marcador cutáneo de infección por VIH, cuando ocurre en pacientes previamente sanos o con psoriasis recalcitrante, y podría ser un marcador de progresión a etapa $\operatorname{SIDA}^{5-8}$. El sarcoma de Kaposi es otro marcador presente en nuestro paciente ${ }^{18}$.

El tratamiento consiste en iniciar terapia antirretroviral asociado a tratamiento tópico o sistémico (fototerapia y/o retinoides orales), dependiendo de la extensión de la psoriasis ${ }^{4}$. La zidovudina (AZT) tiene un efecto antipsoriático, que se podría deber a su interferencia con la síntesis de $\mathrm{ADN}$, disminuyendo la proliferación de queratinocitos. Este efecto es independiente del recuento de linfocitos T CD4 4 . Existen escasos estudios publicados sobre el uso de otros tratamientos sistémicos tales como metotrexato, ciclosporina o agentes biológicos ${ }^{11,15,22,23}$. Estos se encuentran en estudio para evaluar su uso en pacientes infectados por VIH, pero se prefieren por sobre los corticoesteroides sistémicos, por el riesgo de un brote pustular al disminuir tratamiento ${ }^{7,24}$ (Tabla 2).

\section{Conclusión}

Los pacientes infectados por VIH presentan distintas manifestaciones cutáneas. Estos marcadores cutáneos son importantes en la sospecha de infección por VIH, por lo que en presencia de eritrodermia psoriática en un paciente joven siempre se debe sospechar una infección por VIH. El tratamiento consiste en iniciar la terapia antirretroviral.

\section{Resumen}

La infección por VIH puede presentarse con distintas manifestaciones cutáneas, que en algunas ocasiones son consideradas marcadores de infección. Una posible manifestación es la eritrodermia psoriática, que corresponde a 
una forma generalizada de psoriasis. Presentamos un caso clínico de un hombre joven en que se sospechó una infección por VIH por un cuadro de eritrodermia psoriática confirmada por biopsia, asociado a un sarcoma de Kaposi. Posteriormente, la infección por VIH fue confirmada por serología. Se manejó con terapia antirretroviral, con buena respuesta al mes de tratamiento. La eritrodermia psoriática se puede considerar un marcador cutáneo de infección por VIH cuando ocurre en pacientes previamente sanos o con psoriasis recalcitrante.

\section{Referencias bibliográficas}

1.- Joint United Nations Programme on HIV/ AIDS (UNAIDS). Global AIDS update. 2016. Disponible en: http://www.unaids.org/ sites/default/files/media_asset/global-AIDSupdate-2016_en.pdf

2.- Departamento de Epidemiología del Ministerio de Salud (MINSAL). Informe nacional: evolución de la infección por VIH/SIDA, Chile 1984-2012. Rev Chilena Infectol 2015; 32: $17-43$

3.- Ward H A, Russo G G, Shrum J V. Cutaneous manifestations of antiretroviral therapy. J Am Acad Dermatol 2002; 46: 284-93.

4.- Martín A, Omar M, Romero A, Duro G, Hernández J. Psoriasis e infección por VIH. Med Integr 2002; 39: 361-4.

5.- Jordaan H F. Common skin and mucosal disorders in HIV/AIDS. SA Fam Pract 2008; 50: 14-23.

6.- Maurer T A. Dermatologic manifestations of HIV infection. Top HIV Med 2005; 13 : $149-54$.

7.- Navarrete-Dechent, Ortega R, Fich F, Concha $\mathrm{M}$. Manifestaciones dermatológicas asociadas a la infección por VIH/SIDA. Rev Chilena Infectol 2015; 32: 57-71.

8.- Ho K M. Cutaneous manifestations of HIV infection. Hong Kong J Dermatol Venereol 2000; 8: 100-7.
9.- Julià M, Darwich E, Mascaró J M. Psoriasis. JANO 2009; 1748: 15-22.

10.- Sehgal V N, Srivastava G, Sardana K. Erythroderma/exfoliative dermatitis: a synopsis. Int J Dermatol 2004; 43: 39-47.

11.- Grant-Kels J, Fedeles F, Rothe M. Exfoliative dermatitis. In: Goldsmith L, Katz S, Gilchrest B, Paller A, Leffell D, Wolff K, editors. Fitzpatrick's Dermatology in general medicine. 8th ed. New York: Mc Graw Hill; 2012. p. 26684.

12.- Cedeno- Laurent F, Gómez-Flores M, Méndez $\mathrm{N}$, Ancer-Rodríguez J, Bryant J L, Gaspari A A, et al. New insights into HIV-1-primary skin disorders. J Int AIDS Soc 2011; 14: 5.

13.- Rigopoulos D, Paparizos V, Katsambas A. Cutaneous markers of HIV infection. Clin Dermatol 2004; 22: 487-98.

14.- Morar N, Willis-Owen S A, Maurer T, Bunker C B. HIV-associated psoriasis: pathogenesis, clinical features, and management. Lancet Infect Dis 2010; 10: 470-8.

15.- Obuch M L, Maurer T A, Becker B, Berger T G. Psoriasis and human immunodeficiency virus infection. J Am Acad Dermatol 1992; 27 667-73.

16.- Ramos C, Quijano E, Carayhua D, Sánchez R. Psoriasis en pacientes con VIH: presentación de nueve casos. Rev Soc Peruana Dermatol 2011; 21: 154-7.

17.- Blanco González O, Larrondo Muguercia R,
Blanco González B, Rodríguez Barreras M. Psoriasis y SIDA: reporte de 2 casos. Rev Cubana Med Trop. 2000; 52: 148-9.

18.- Garza-Garza R, González-González S, Ocampo-Candiani J. Manifestaciones cutáneas del VIH. Gac Med Mex 2014; 150 Suppl 2: 194-221.

19.- Sterry W, Steinhoff M. Erythroderma. In: Bolognia J, Jorizzo J, Schaffer J, editors. Dermatology. 3rd ed. New York: Elsevier Saunders; 2012. p. 171-81.

20.- Zip C, Murray S, Walsh N M. The specificity of histopathology in erythroderma. J Cutan Pathol 1993; 20: 393-8.

21.- Tomasini C, Aloi F, Solaroli C, Pippione M. Psoriatic erythroderma: a histopathologic study of forty-five patients. Dermatology 1997; 194: 102-6.

22.- Castro M K D, Jurado S C F, Martínez E V. Eritrodermia como primera manifestación de psoriasis. Comunicación de un caso, en una paciente joven manejada satisfactoriamente con metotrexato. Rev Cent Dermatol Pascua 1999; 8: 96-100.

23.- Piqué-Durán E, Pérez-Cejudo J. Eritrodermia psoriásica tratada con etanercept. Actas Dermosifiliogr 2007; 98: 508-10.

24.- Boyd A, Menter A. Erythrodermic psoriasis: Precipitating factors, course, and prognosis in 50 patients. J Am Acad Dermatol 1989; 21 : 985-91. 